\title{
Decentralising the Digital Rights Management Value Chain by means of Distributed License Catalogues
}

\author{
B. Vassiliadis, V. Fotopoulos, A.N. Skodras \\ Digital Systems \& Media Computing Laboratory, School of Science and \\ Technology, Hellenic Open University, \\ 13-15 Tsamadou st., GR-26222, Patras, Greece \\ $\{b b, v f o t o p 1, s k o d r a s\} @ e a p . g r$
}

\begin{abstract}
Digital Rights Management (DRM) systems' interoperability is becoming one of the main obstacles for their wider adoption, especially from medium and small size users. Interoperability issues affect, among others, the management of content usage rules by third parties (authorities) and the automation of licensing procedures upon the purchase of digital content. The fundamental question of who is handling content licenses in the national or global DRM value chain is complex, with business, social and technological extensions. In this paper, we discuss current trends in DRM systems technology and business modelling and briefly present a proposal for handling digital content licensing, Distributed License Catalogues (DLCs). The DLC concept, borrowed from web engineering, makes available ("advertises") content or services concerning DRM functionalities, enabling multi-party DRM eco-systems.
\end{abstract}

\section{Introduction}

Digital Rights Management (DRM) systems appeared as a natural consequence of the need to electronically manage a wide range of IPR (Intellectual Property Rights) functionalities such as description, identification, trading, protection, monitoring and tracking. These functionalities should be able to be applied over both tangible and intangible assets including rights workflow and rights owner relationships [1].

DRMs are complex information systems in the sense that they highly involve technological, economical, business and most importantly, social factors. The first generation of DRM business models, as defined by the Imprimatur project [2] and later by Ianella's vision of their information architecture [3], was offering a full range of functionalities. The first DRM implementations showed the potential of the technology but also some pitfalls of the complex, full functionality approach.

Please use the following format when citing this chapter:

Vassiliadis, Vassileios, Fotopoulos, Vassileios, Skodras, Athanassios, 2006, in IFIP International Federation for Information Processing, Volume 204, Artificial Intelligence Applications and Innovations, eds. Maglogiannis, I., Karpouzis, K., Bramer, M., (Boston: Springer), pp. 689-696 
Immature economics, lack of standards, potential misuse of user rights, prohibiting costs for small users, lack of appropriate legislation are some of the problems that need to be solved next. These primary issues naturally imposed an interoperability gap between different DRM implementations. This problem cannot be attributed solely to technology (e.g. lack of standards) but to other factors as well. For example, a social factor is that users are not satisfied with existing, strict usage rights policy of current DRM solutions [4,5]. The lack of relevant legislation, which is not matured in many countries, and usability are also drawbacks for DRM's wider adoption [6]. Most importantly, full functionality solutions are too costly for medium and small users

DRM is a relatively new technology, and as such it also faces a definition problem; there is no clear agreement of what DRM is "in the whole" [7]. The first days of the Internet saw a similar problem. The lack of standards caused a misuse of the term. Only when standards and architectures were placed, the Internet took the form we all know and use today.

DRM systems' interoperability is becoming one of the main obstacles for their wider adoption, especially from medium and small size users. Interoperability issues affect, among others, the management of content usage rules by third parties and the automation of licensing procedures upon the purchase of digital content. One of the fundamental questions in the DRM business model is "who is handling content licenses in the national or global DRM value chain?". Content usage rules are provided by the creators/owners. The classic business model does foresee many authorities who are responsible for supervising the proper use of usage rules, however technological, political, social, legislative issues are not dealt-with in such multi-authority environments. Does a single authority guarantees equal management of rights? Should all the functionalities of an authority (licensing, monitoring and reporting) be handled by one organization or could they be provided by many? Should the DRM community learn from other similar endeavours? For example, the most successful on-line, single-authority model is the DNS address system. It has been successful in managing Internet addresses for some time now, but the managing organization, a US-based institution, is getting increased criticism from abroad of the type: "why should a foreign institution manage our internet addresses?". Digital rights are equally important; governments would like at least some control over the management of the national content. Similarly, professional associations could also claim the role of authorities. The same goes for private organizations.

Having many authorities in the DRM value chain may possibly become a real need in the future, a need that will certainly create a whole lot of new questions of business, social and technological extensions. In this work, we examine a DRM business model where many authorities are handling digital content licensing. Discovery mechanisms are a critical factor to this distributed model. Its wider adoption requires addressing, among others, problems such as publication, description, discovery and security. We address the discovery issue by means of Distributed License Catalogues (DLCs). DLC is based on a web engineering concept, and more particularly UDDI (Universal Description, Discovery and Integration) catalogues, a standard for publishing and discovering web services [8]. We propose a new DRM business model where content providers or associations may act as authorities using thematically-oriented directories, to publish licenses and 
content rights. Although these catalogues are distributed, their architecture can be either client-server or Peer to Peer.

The rest of the paper is structured as follows: section 2 briefly describes current technology trends in DRM systems and presents the basic DRM business model. Section 3 introduces DLCs while section 4 briefly discusses possible implementation architectures. Finally, conclusions are drawn in section 5 .

\section{Digital Rights Management systems}

Complete solutions to IPR protection and management such as DRM systems have been proposed for the management of licenses of media content throughout its lifecycle [9]. Technologically, the area of DRM is unique in the sense that it involves many diverse sub-areas: cryptography, signal processing and information theory, ecommerce, business modelling, legal and social aspects just to mention a few. Current DRM systems are complicated, expensive and inherit many of the shortcomings of the methods they use. They are considered however by many, a solution of great prospect.

A DRM system provides a complete set of functionalities for managing IPR [7]. DRMs can either be stand-alone systems or part of a larger on-line selling system relying on licenses which specify the content usage rules. Content is distributed with or without licenses but it cannot be used without them. Rules can be attached, embedded to the content or delivered independently [10]. It is important to note that DRM is about digitally managing rights and not managing digital rights; modern DRM systems cover the full range of IPR management including the description, identification, trading, protection, monitoring and tracking of all forms of rights' usage $[3,11]$.

In [12] the authors discuss two definitions for DRM systems, the narrow and the broad. The narrow definition refers to systems that persistently protect content using mainly encryption techniques. The digital content is packaged (encrypted and metadata enriched) and then provided through distribution channels. Users need special controllers (client side $\mathrm{s} / \mathrm{w}$ ) in order to be authenticated and gain access through the decryption of content. License servers may be used to manage licenses describing access rights and conditions. The broad definition includes the abovementioned functionalities and further extends rights management. It includes definition, management and tracking of rights (business rights, licensing, access tracking etc.).

Recent attempts to deploy DRM systems have shown that their success depends not only on technology but business issues as well. The underlying business model, that is the mechanism by which a business intends to generate revenue and profits, is of paramount importance. The business model defines the plans to serve customers involving both strategy and implementation. It greatly affects, and is affected, by the technology used. The creator produces the digital content and provides the usage rules to a third party (authority) which is responsible for supervising its proper use. Distributors receive the content from the creators and distribute it through the appropriate channels (e.g. e-shops) to the end-users (buyers). In order for the buyer 
to use the content, the appropriate license must be obtained by the authority. This happens after the appropriate request is sent to the authority by the buyer. The transaction is concluded when the authority pays royalties to the creator [2]. There is a plethora of DRM payment models: pay as you use, try-first buy-later, pay-per-view etc. Payment rules are closely connected to the way the content is supposed to be used and as such they are described by rules.

\section{Supporting many Authorities with DLCs}

Suppose that in the, not so distant, future the national or international legislation permits associations, companies or even single users to act as authorities for content they have created or they own. Although it is obvious that most of these authorities would not be able to provide the full functionality of an authority as described in the previous section, they could provide at least some kind of licensing services and accept payments (through integrated e-banking solutions). Overlooking the huge political, cultural and social implications which are not within the scope of this work to analyze, the question of the most efficient technology that will support such a model, naturally arises.

Traditionally, network applications have relied on client-server as their core technological model. Classic DRM designs of the past decade rely on such a model especially when dealing with Authority-related transactions. But what happens when authorities are dispersed in cyberspace and are heterogeneous in their interface configurations? In such a case users should be equipped with agents capable of performing Application-to Application (A2A) operations in order to ease interaction with different providers and authorities. Creators and distributors will be able to cooperate with different Authorities. The peers of such a network will be able to provide different services (functionalities) of a DRM system on-line. This model resembles more to the Service-Oriented [13] or, somewhat lesser, to the Peer to Peer [14] computing models than the client-server one (figure 1).

Service-Orientation supports the development of applications as if they were a connected network of functionalities (services) available, in a network-enabled environment, within and across different organizations. Services, the building blocks of Service-Oriented systems, may be made public, searched, reused and combined to form complex business processes while in the same time retaining a significant level of flexibility. This computing model will hopefully enable the transition of the software industry into a service industry.

Among the many technological questions that need to be answered in such a configuration is how to implement an efficient discovery mechanism, a critical factor to the overall utility of the scheme. Since there are many Authorities, Creators and Distributors on-line along with numerous digital content repositories, how can a user find the appropriate service/license/content? The same question was posed to the Web Engineering community when different software vendors made available online their web services. Web service registries are helping to narrow down the negotiation and searching time needed for service discovery. Their basic concept lays in the matching mechanism of the contractual and technical profile of the query 
to that of the services. In order for the searching procedure to be fast and more importantly automatic, information needs to be machine-processable. At present, Web services are mainly advertised in catalogues which are based on the Universal Description, Discovery and Integration standard - UDDI [8]. UDDI has become the predominant technological environment for service discovery. A large number of such centralized registry/repository implementations for intra-enterprise communication, each focusing on registering services of interest to respective groups, are anticipated. Besides UDDI, decentralized approaches based on Peer-toPeer have been proposed in order to achieve increased fault-tolerance behaviour [15].

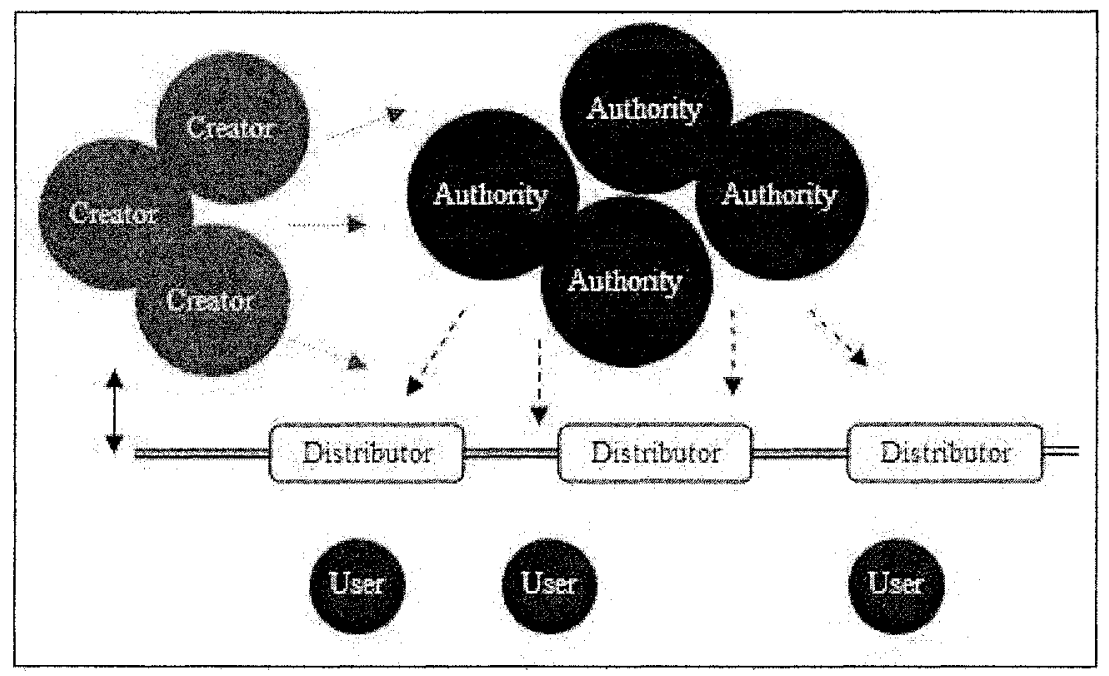

Fig. 1. A multi-party DRM eco-system

In this work we propose a similar conceptual approach. Among other services, digital content licensing is supported by Distributed License Catalogues (DLCs). DLCs are catalogues, actually registries or indexes that make available ("advertise") content or services concerning DRM functionalities, interfaces, creators, pricing etc. A DLC may be used by different nodes (creators and authorities alike) hosting a total DRM solution or providing a subset of DRM functionalities. New DRM business models can be supported where content providers or associations may act as authorities using centralized and thematically-oriented directories, to publish licenses and content rights. The structure of these registries should follow some kind of a standard following the example of UDDI. 


\section{The DLC Architecture}

The problem facing the multi-party DRM eco-system is that each node (organisation) probably provides a different (or slightly different) set of monitoring and management services for its content. This diversity in services that actually provide the same core functionality (e.g. user tracking) is a natural consequence of the diversity between organisations. For example, different corporate culture and strategic goals may lead to different implementations of asset management services. Diversity stems from the diversity of media and metadata types used also; different representation of media artefacts include structured documents, still or moving images, audio and 3D or virtual reality objects. Representation is closely connected to the encoding method used for compressing digital data. New compression standards such as JPEG2000 and MPEG4 offer many build-in IPR management characteristics that modern DRM systems (and their respective services) should be able to exploit. Therefore, each node (or sets of nodes) in the aforementioned distributed scheme should provide a version of some of the core services. Core services can be coordinated using workflow management to build a larger, more complicated service. Services may also use digital artefacts from different repositories; for example, a user designs a multimedia presentation using digital still images from node $\mathrm{A}$ and small digital video clips from node $\mathrm{B}$. DLCs publishing services and information of nodes should provide the starting point for such complex operations.

The DLC concept is about publishing service and artefact information and not about organisation and storage. For this reason it uses only semantics, that is metadata descriptions for services and artefacts and one multilayer ontology scheme for cross-searching like the one proposed in [16]. The DLC sits on top of the storage implementation giving birth to a 4-tier model, in contrast to the 3-tier models of web systems (figure 2). The DLC metadata are used to describe license information, media artefacts descriptions and available services from the current vendor using a UDDI-like structure. The multi-layer ontology scheme is used, on one hand for faster local searching and on the other, for facilitating service/content composition using information from other nodes. It can also be effectively used when a node is comprised by a set of sub-nodes (e.g. in the case of a portal). Similarly to the approach described in [16], our approach introduces a three layer semantic description of node contents (i.e. digital artefacts and services): the Upper Ontology layer, describes the basic concepts of the domains of knowledge of the content/services, a set of description ontologies (Domain layer) represents a more detailed description of each domain and the Semantic layer where the different semantic description of the cooperating nodes or sub-nodes lays. The proposed indexing scheme includes additional mapping information between the ontologies in the three layers providing the necessary information to search engines in order to navigate inside the ontology-based index.

Similar to web service discovery, we foresee two approaches for utilising the distributed DLC architecture: centralised and decentralised. In the centralised approach (actually the client-sever model) services, content or information about the content (usage rights) are registered in the DLC repository and clients are able to 
search it in order to find the appropriate solution. Unlike UDDI, the information in DLCs should not be made public; only authenticated users should gain access to information. A multi-level security scheme should provide different access rights to different users. In general, the client-server model suffers from performance bottlenecks when too many users search the same registry at the same time. The classic approach to add more servers or use load balancing techniques is not an efficient (or cost effective) solution. Decentralised approaches for service and information discovery have already been proposed by large software vendors. Approaches based on P2P infrastructures are gaining popularity in the Web Engineering field. For example, decentralized Web Services discovery uses either a structured or unstructured $\mathrm{P} 2 \mathrm{P}$ infrastructure, although some hybrid solutions have appeared as well (e.g. super-peers). Unstructured P2P systems like Gnutella, define neighbours of peers in an ad-hoc manner and as such, they are appropriate for highly-transient peer populations communication [14]. Due to the lack of any structure, location mechanisms face significant problems relating to availability, scalability and persistence. Nevertheless, peers enjoy a large degree of autonomy. Similarly, discovery mechanisms in distributed DRM models may rely on P2P infrastructures reducing bottleneck problems.

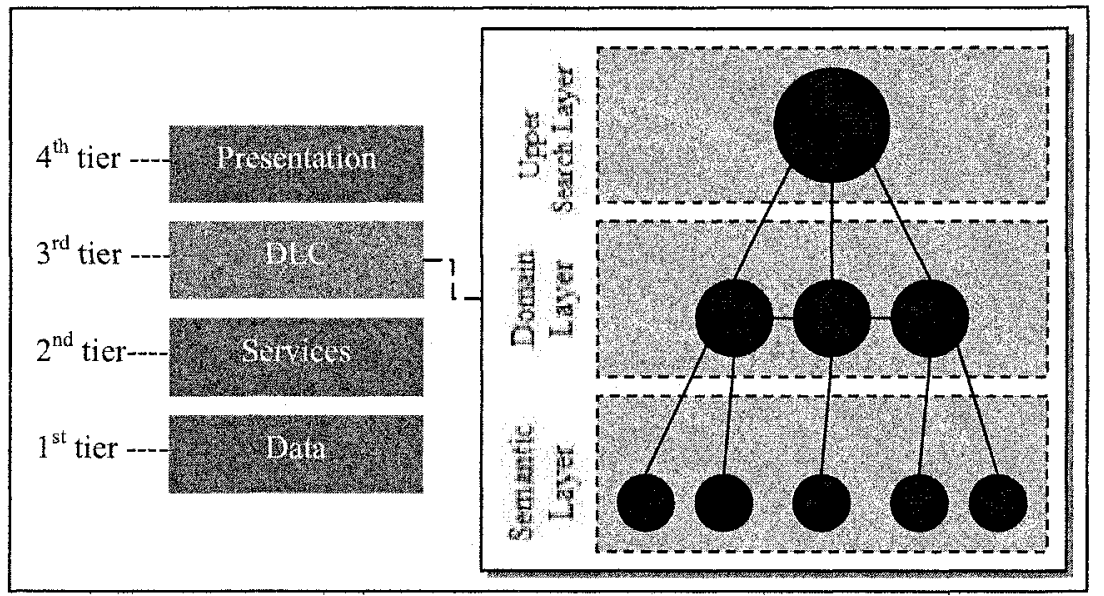

Fig. 2. 4-tier node architecture and a snapshot of a DLC multilayer ontology scheme.

\section{Conclusions}

The need for Digital Rights Management systems is greater than ever as copyright protection is becoming an important issue for organizations that create, use and distribute digital content through e-commerce channels. This work provided a general discussion of current DRM technological trends and pin-pointed some problems arising at a social, political and business level from their use. A new concept, namely DLCs were proposed as a solution for managing a highly 
distributed ecosystem of creators, authorities and users. DLCs were described here at a conceptual level and many details need to be determined even before deciding if they are a viable solution. However, the main idea behind this concept is to move beyond monolithic architectures that are inflexible and costly, towards ServiceOriented DRM systems.

\section{Acknowledgements}

This work was funded by the European Social Fund, Operational Programme for Educational and Vocational Training II (EPEAEK II), programme Pythagoras (contract no. 89188).

\section{References}

1. Eskicioglu, E.M. (2003). Protecting Intellectual Property in Digital Multimedia Networks. Computer, 36, 39-45.

2. Imprimatur (1999). Imprimatur Business Model, Version 2.1. [on line] http://www.imprimatur.net.

3. Iannella, R. (2001). Digital Rights Management (DRM) Architectures. D-Lib Magazine, $7(6)$.

4. McCalman, P. (2005). International diffusion and intellectual property rights: An empirical analysis. Journal of International Economics, 67(2), 353-372.

5. Towse, R. (2005). Economics and copyright reform: aspects of the EC directive. Telematics and Informatics, 22, 11-24.

6. Beute, B. (2005). Mobile DRM-usability out of the door?. Telematics and Informatics, 22 , $83-96$.

7. Koenen, R.H., Lacy, J., MacKay, M., Mitchell, S. (2004). The long march to interoperable Digital Rights Management. Proceedings of the IEEE, 92(6), 883-897.

8. OASIS (2005). UDDI [on line] http://www.uddi.org/

9. Memon, N., Wong, P.W. (1998). Protecting Digital Media Content. Communications of the $\mathrm{ACM}, 41(7), 34-43$.

10.Cohen, J.E. (2003). DRM and Privacy. Communications of the ACM, 46(4), 46-49.

11.Hwang, S.O., Yoon, K.S., Jun, K.P., Lee, K.H. (2004). Modelling and implementation of digital rights. Journal of Systems and Software, 73(3), 533-549.

12.Rosenblatt, B., Trippe, B., Mooney, S. (2002). Digital Rights Management - Business and Technology. M\&T books.

13.Singh, P.M., Huhns, M.N. (2005). Service Oriented Computing, Semantics, Processes, Agents. Wiley Press.

14.Androutsellis-Theotokis, S., Spinellis, D. (2004). A Survey of Peer-to-Peer Content Distribution Technologies. ACM Computing Surveys 36(4), 335-371.

15.Sakkopoulos E., Makris Ch., Sioutas S., Triantafillou P., Tsakalidis A., Vassiliadis B. (2005). NIPPERS: Network of InterPolated PeERS for Web Service Discovery. IEEE International Conference on Information Technology: Coding and Computing, 193-198.

16.Alexakos, Ch., Vassiliadis, B., Votis, K., Likothanassis, S. (2005), A Multilayer Ontology Scheme for Integrated Searching in Distributed Hypermedia. International Workshop on Adaptive and Personalized Semantic Web (in conjunction with ACM Hypertext 2006). [on line] www.ru5.cti.gr/HT05/files/likothanasis.pdf 